\title{
ORIGINAL ARTICLE \\ The natural course of spinal cord injury: changes over 40 years among those with exceptional survival
}

\author{
JS Krause ${ }^{1}$, JC Newman ${ }^{2}$, JMR Clark ${ }^{1}$ and M Dunn ${ }^{3}$
}

Objectives: To identify 40-year longitudinal changes in health, activity, employment, life satisfaction and self-rated adjustment after spinal cord injury.

Study design: Longitudinal, mailed self-report.

Methods: Participants were identified from outpatient records of a Midwestern USA university hospital in 1973. Follow-ups were conducted in 1984 and approximate 10-year intervals thereafter. A total of 49 participants completed each of the five assessments. Data were reviewed and analyzed by research team members and a research associate with experience in biostatistics at a medical university in Southeastern USA. Life Situation Questionnaire included the following: (1) demographic and injury characteristics, (2) educational status and employment, (3) community participation, (4) life satisfaction, (5) adjustment, and (6) recent medical history.

Results: Proportion of individuals with $10+$ non-routine physician visits increased from consistently $<10 \%$ to $>40 \%$ during the 40 years. Proportion who spent a week or more in hospital increased from a low of $10 \%$ at 20 -year follow-up to $43 \%$ at 40 -year follow-up. Percentage employed and average hours employed initially improved over time but decreased substantially during the last two times of measurement. Satisfaction with health, sex life and social life declined over time, whereas satisfaction with employment improved initially and was maintained over time. Self-rated current adjustment remained stable, whereas predicted future adjustment declined steadily over 40 years.

Conclusions: Age-related declines were apparent for need of physician visits and hospitalizations, with notable declines in satisfaction with sex life, social life and health. However, not all indices declined over time. Participants appeared to maintain stability when rating their own adjustment.

Spinal Cord (2017) 55, 502-508; doi:10.1038/sc.2016.159; published online 6 December 2016

\section{INTRODUCTION}

Individuals with spinal cord injury (SCI) face significant long-term challenges to health, psychological well-being and longevity. ${ }^{1-3}$ Life expectancy is substantially reduced, the extent of which depends on the level and neurological completeness of injury. ${ }^{4}$ Yet, many individuals live 40,50 or more years after SCI onset, substantially exceeding what is typical for those with SCI. Groah et al. ${ }^{5}$ highlighted the need for longitudinal studies of aging and SCI to help us understand the issues faced by those who reach aging milestones with SCI. To accomplish this, we must study those who have reached the greatest milestones, including those who have substantially outlived their life expectancy.

Longitudinal studies have provided insight into physical health, psychosocial outcomes and functional changes over time in individuals with SCI. Hitzig et al. ${ }^{6}$ examined premature aging in bodily systems in those with SCI and reported evidence of premature aging in the musculoskeletal and respiratory systems as suggested by a systemic review of longitudinal literature. A 17-year longitudinal study ${ }^{7}$ identified a significant increase in the frequency of cardiometabolic syndrome over time, with Hispanics having significantly higher frequency. Other longitudinal examinations have suggested improvement in self-reported constipation, bladder stones, rectal bleeding and urinary tract infections over time following SCI. ${ }^{8,9}$

Psychosocial outcomes have been examined, with longitudinal evaluations of depressive symptomatology following injury suggesting sustained rates of probable major depression in the first 5 years following injury. ${ }^{10}$ Other studies suggested increased depressive symptomatology over longer follow-up periods. ${ }^{9,11}$ In persons with long duration SCI (at least 20 years after injury), declines in morale and life satisfaction assessed over 10 years have been noted. ${ }^{9,12}$ Studies have provided mixed findings of social integration, again in those with long duration injuries, with one suggesting improvement ${ }^{9}$ and others indicating a decline over approximately 10 years. ${ }^{12,13}$

The SCI Longitudinal Aging Study was initiated by Nancy Crewe and associates in 1973 and was developed as a means of understanding the life situation of people with SCI living in the community. Although not initially designed as a longitudinal study, a follow-up was conducted approximately 11 years later. ${ }^{14}$ Several favorable changes were noted, including an increase in the employment rate, increased satisfaction with employment and improved self-ratings of overall

${ }^{1}$ Department of Health Sciences and Research, Medical University of South Carolina, Charleston, SC, USA; ${ }^{2}$ Department of Medicine, Medical University of South Carolina, Charleston, SC, USA and ${ }^{3}$ Mountain View, CA, USA

Correspondence: Dr JS Krause, Department of Health Sciences and Research, Medical University of South Carolina, 151-B Rutledge Avenue, Suite B102, Charleston, SC 29425, USA.

E-mail: krause@musc.edu

Received 13 April 2016; revised 7 June 2016; accepted 10 June 2016; published online 6 December 2016 
adjustment over time. The number of hospitalizations and days hospitalized (within 24 months prior to assessment) decreased over the 11-year interval. A survivor effect was observed, however, where individuals with better psychosocial and vocational adjustment were more likely to survive to follow-up. ${ }^{15}$ Therefore, the primary trends were improving outcomes over time among survivors, with less well-adjusted participants having a greater likelihood of mortality.

The same overall trend for improvements was generally maintained over subsequent 4-5-year intervals, although a new pattern emerged by the 30-year follow-up. ${ }^{16}$ Sitting tolerance, years of education, employment rates and satisfaction with employment and adjustment improved in the first 15 years after enrollment. Although these changes tended to remain stable during the next 15 years, other outcomes declined. Sitting tolerance decreased from 15 to 30 years, and satisfaction with social life and sex life also declined. There was measurable increase in the number of non-routine physician visits. Employment outcomes improved over the course of the study, ${ }^{16-20}$ with findings suggesting the greatest improvement in the initial 15 years following study initiation. ${ }^{16,17}$

Further changes were observed by the 35 -year follow-up. Although no changes in the frequency of weekly visitors and outings were observed in the first 20 years, ${ }^{18,19}$ there were significant declines in these proctors of community participation at 25 years and 35 years following initial data collection. ${ }^{17,20}$ Further decreases in satisfaction with social life, sex life and general health were noted, particularly in the latter follow-ups. Non-routine physician visits increased over time. Following an initial decrease, frequency of hospitalizations and days hospitalized plateaued during these latter follow-ups. ${ }^{16-20}$ The survivor effect continued to be observed. ${ }^{21-23}$

In summary, there appears to be a mixed pattern of changes within the natural course of aging with SCI, with improvements noted in the first 15-20 years after enrollment but with some changes thereafter. Participation, health and satisfaction appear to be most likely to decline among survivors, whereas employment is most likely to be maintained. As there is a significant survivor effect, the findings for long-term survivors become less representative of the full spectrum of people with SCI but more directly relevant to those reaching or exceeding their life expectancy. Studies of those with exceptional survival of $\geqslant 40$ years are rare.

\section{Purpose}

The purpose of this study was to explore the natural course of aging after SCI by identifying changes in participation, employment, health and quality of life outcomes over 40 years, building upon the previous 35 years of research. We assess changes in outcomes over 10-year intervals. The unique contribution is the consistent measurement of outcomes over 40 years and our ability to identify changes in outcomes over that length of time. The findings help us understand the natural course of SCI among those who have had extraordinary survival ( $>40$ years after SCI onset) and gain a glimpse of the future and the unique problems that have developed over time, even among those who have outlived their expectation of survival.

\section{MATERIALS AND METHODS}

\section{Participants}

The SCI Longitudinal Aging Study initially enrolled a cohort of 256 participants, with 49 participants remaining in the study 40 years later. The inclusion criteria were: (1) traumatic SCI, (2) at least 2 years since SCI onset, and (c) a minimum age of 18 years at study enrollment. Although neurological completeness of injury was not measured, all individuals had significant residual impairment, sufficient to require SCI services. Participants were drawn from a population of all possible cases who met the selection criteria from outpatient records at a USA Midwestern university hospital and a rehabilitation hospital in Minnesota.

The response rates have been strong throughout the course of the study, with an initial $85 \%$ response rate in 1973. At the time of the first follow-up in 1984, 154 participants remained in the study, 46 were deceased, 33 could not be located and 23 chose not to participate. The effective response rates thereafter have been outstanding, although attrition ultimately reduced the number of participants to 49 at the 40 -year follow-up, largely owing to mortality. A more detailed reporting of study procedures, response patterns and measures appears elsewhere. ${ }^{24}$

\section{Sample characteristics: 40-year follow-up}

All participants were white, and $85.7 \%$ were male. There was a mean chronological age of 64.9 (s.d. $=5.0$ ) years and mean years after injury of 45.9 ( $\mathrm{sd}=4.1$ ). Just over half $(55.1 \%)$ had cervical injuries. In terms of neurological completeness, $53.9 \%$ reported sensation below the level of lesion, and $27.7 \%$ reported voluntary movement. Functionally, only $10.4 \%$ reported they could walk at all. They were well-educated (mean years 15.6; s.d. $=2.8$ ). For marital status, $59.2 \%$ were married, $16.3 \%$ were divorced, $16.3 \%$ never married and $8.2 \%$ were widowed or a member of an unmarried couple.

\section{Data collection procedures}

Self-report measures have been obtained by mail during each time of measurement. The data collection protocol has been constant over the years. Cover letters explaining the study have routinely been sent prior to sending a full packet of materials, followed by two subsequent mailings and a phone call to non-respondents. Additional mailings are used if the person has lost or discarded their materials but continue to express an interest to participate.

Extensive efforts have been made to locate participants, including using previous addresses, contact information for relatives and directory assistance. In recent years, we have used available internet mechanisms. Participant remuneration was provided beginning in 1989 and has increased over the years from $\$ 5$ to $\$ 40$. Although no remuneration was provided in 1985 , participants were offered copies of Employment Following Spinal Cord Injury, an employment handbook developed from information gathered during the first assessment. ${ }^{25}$

\section{Measures}

The Life Situation Questionnaire was designed specifically for the study in 1973 to obtain mostly objective, verifiable information about the overall psychosocial, vocational and medical outcomes of people with SCI. The initial version of the Life Situation Questionnaire included the following content areas: (1) demographic and injury characteristics, (2) educational status and employment, (3) community participation, (4) life satisfaction, (5) adjustment, and (6) recent medical history (activities of daily living and instrumental activities of daily living were also measured but not reported in this study).

Demographic characteristics, including sex, age and marital status, were collected. Marital status was defined as single, married or separated/divorced/ widowed.

Injury characteristics in the original Life Situation Questionnaire were limited to injury level (cervical versus non-cervical) and date of injury. Years after injury was calculated from the date of injury and reported continuously.

Educational status was measured by the number of years of education.

Employment history was measured by current employment status (working yes/no) and hours per week working.

Participation was measured by three general items including: sitting tolerance $(0-7,8-12,>12 \mathrm{~h})$, frequency of weekly social outings and frequency of weekly visitors.

Psychosocial adjustment was measured initially with two sets of items. These included six life satisfaction items measured by a five-point Likert scale ( $1=$ very dissatisfied; $5=$ very satisfied $)$ that included the following: living arrangements, employment, finances, social life, sex life, and general health. The second set of items were two self-rated adjustment scales, each of which asked participants to rate their adjustment on a 10-point ladder from worst possible 
Table 1 Comparison of respondents and drop-outs using baseline characteristics

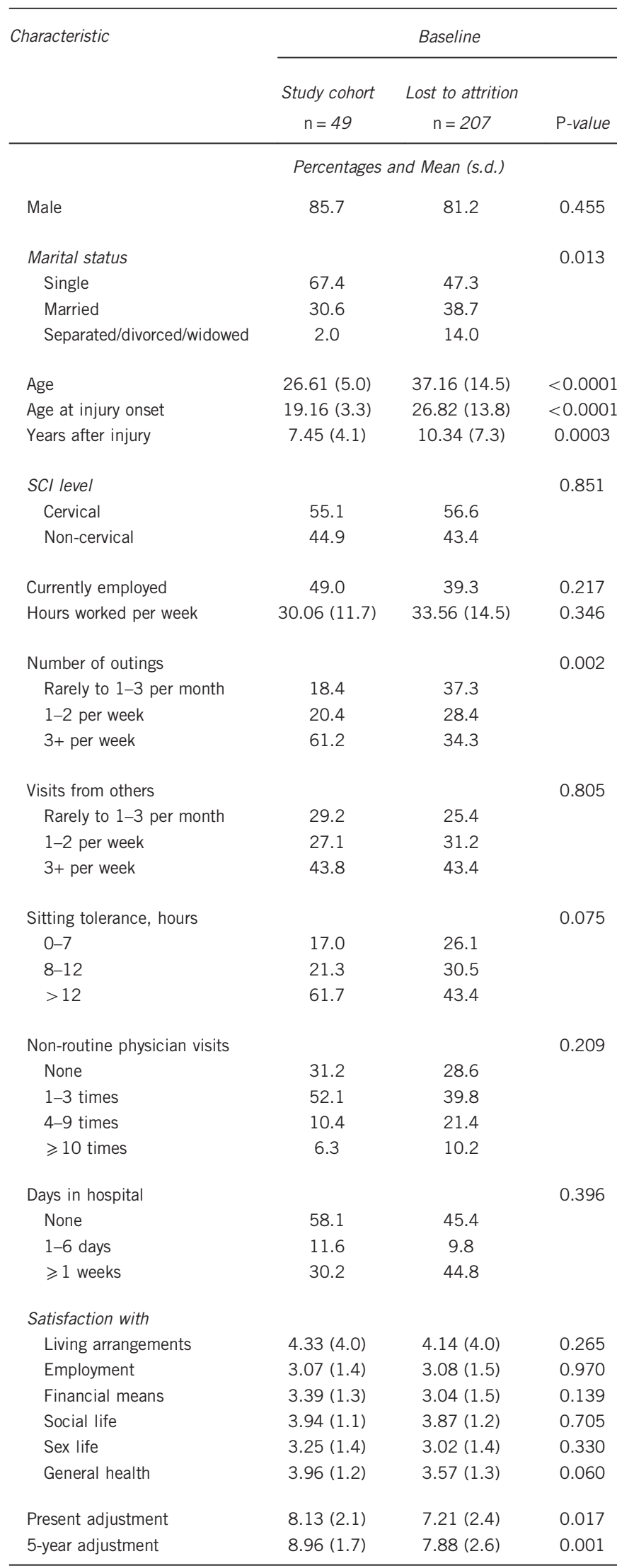

Abbreviation: $\mathrm{SCl}$, spinal cord injury. Values are presented as percentages or mean (s.d.).
(1) to best possible (10). The two items were current adjustment and future adjustment in 5 years.

Measurement of medical history included non-routine physician visits (none, 1-3 times, 4-9 times, $\geqslant 1$ weeks) and days hospitalized (none, 1-6 days, $\geqslant 1$ weeks). Both measures asked participants to report this information within the 24 months prior to the assessment.

\section{Statistical analysis}

Descriptive statistics were calculated for the study cohort, and participants were lost to attrition at all time points. Values were reported as frequency in percentages for categorical variables or means and s.d. for continuous variables. Differences between the two groups were analyzed using Student's $t$-test for continuous measures and Chi-square or Fisher's Exact tests for categorical variables. Frequencies for attrition by educational attainment were not reported at baseline owing to a large proportion of missing values. Mean values were imputed for minimal missing data in continuous measures. The sample cohort of 49 participants included participants who had completed surveys at all five time points. Generalized linear mixed models for repeated measures were performed to assess temporal trends over the five times of measurement (40 years). Several covariance structures were tested, and the autoregressive order 1 covariance structure was ultimately selected for the models. All analyses were performed using SAS v.9.4. A $P$-value of $\leqslant 0.05$ was considered statistically significant.

\section{Statement of ethics}

We certify that all applicable institutional and governmental regulations concerning the ethical use of human volunteer were followed during the course of this research.

\section{RESULTS}

\section{Attrition}

There were several significant differences in characteristics and outcomes at baseline (1973) between those who participated throughout the study and those who dropped out at some point (Table 1). Those who dropped out were, on average, older in age, older at injury onset, had more years after injury and were more likely to be married or separated, widowed or divorced. They also left their homes less frequently and reported lower current and predicted future adjustment scores. None of the following characteristics were significantly different between respondents and those who dropped out: sex, injury level, employment status, visits from others, sitting tolerance, non-routine physician visits, days in the hospital, or the six satisfaction items.

\section{Comparisons over time}

Employment and participation. The proportion of individuals employed changed significantly over time, increasing from $49.0 \%$ at baseline to a high of $71.4 \%$ at the 20 -year follow-up but with a substantial decline from $68.8 \%$ at the 30 -year follow-up to $27.1 \%$ during the 40-year follow-up (Table 2). The number of hours working per week among those who continued to be employed also changed significantly over time from a low average of $30.1 \mathrm{~h}$ at baseline to a high of $41.4 \mathrm{~h}$ at the 20 -year follow-up. Hours declined to $31.1 \mathrm{~h}$ per week at the 40-year follow-up. None of the three activity items changed significantly over time.

Utilization of medical services. Significant temporal changes were noted for both non-routine physician visits and hospitalizations. For non-routine physician visits (Figure 1), the proportion reporting none dropped rather dramatically over the five time points from $31.2 \%$ at baseline to $8.3 \%$ at the 40 -year follow-up. Conversely, the proportion reporting $\geqslant 10$ physician visits in the 24 months prior to assessment varied from a range of $4.1-6.4 \%$ over the first four times of 
Table 2 Longitudinal changes over time from baseline to 40 -year follow-up

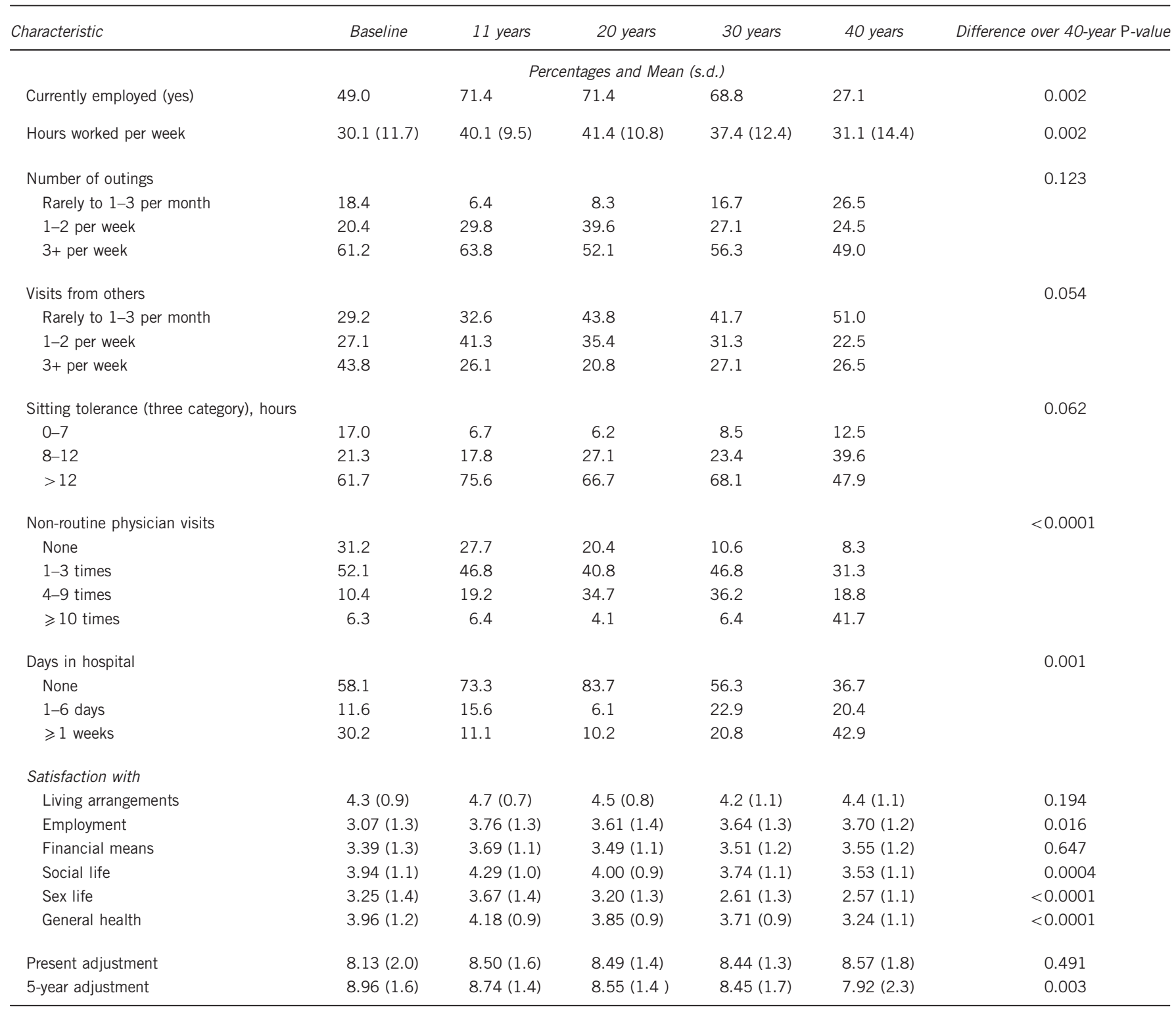

Values are presented as percentages or mean (s.d.).

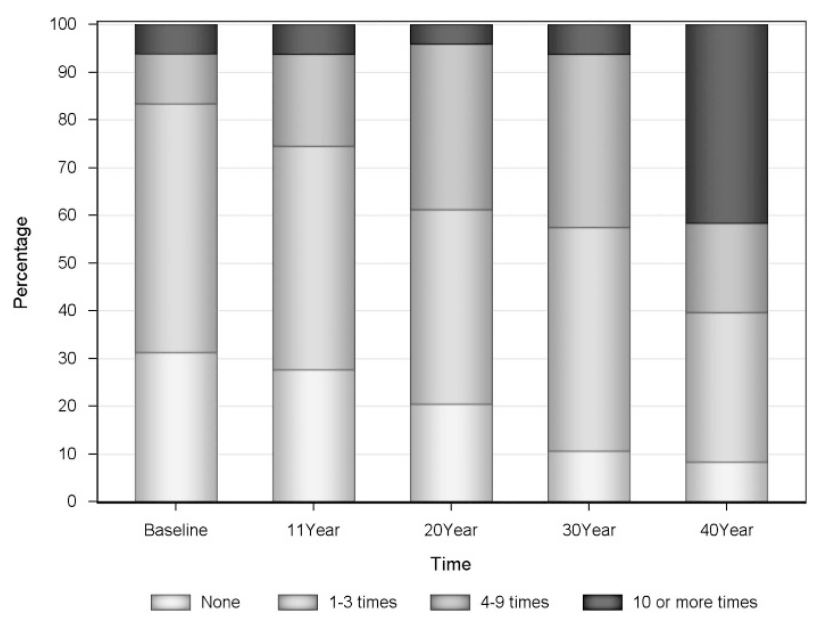

Figure 1 Number of physician visits by time. measurement to $41.7 \%$ at the 40 -year follow-up. Days hospitalized followed a somewhat different pattern (Figure 2), as the proportion of individuals spending $\geqslant 1$ week in the hospital decreased initially from $30.2 \%$ at baseline to a low of $10.2 \%$ at the 20 -year follow-up but increasing during each of the next 10 years to $20.8 \%$ and $42.9 \%$, respectively, creating a upward parabolic trend.

Satisfaction and adjustment. There were significant differences across multiple satisfaction and adjustment measures, including satisfaction with employment, social life, sex life, general health and 5-year adjustment (Figure 3). With the exception of satisfaction with employment, in which there was an increase after baseline that was sustained across the subsequent four times of measurement, each of the other three satisfaction indicators indicated a slight increase initially at 11 years and then gradually declined thereafter. Satisfaction with general health particularly dropped between the last two times of measurement. Ratings of future adjustment declined significantly and consistently over time, although current adjustment remained 


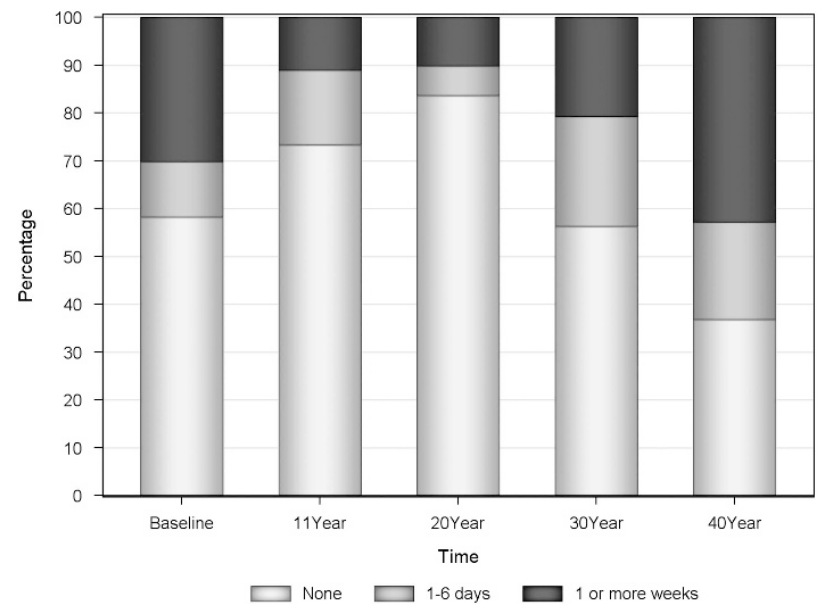

Figure 2 Number of days in the hospital by time.

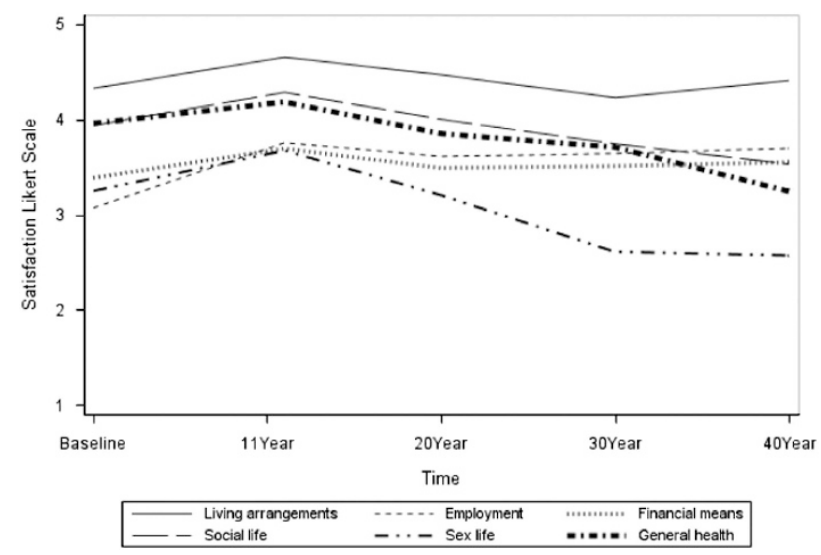

Figure 3 Satisfaction with aspects of life over time.

generally stable. The ratings crossed and inverted at about 30 years after injury, at which point predicted future adjustment was less than that of current rated adjustment (Figure 4).

\section{DISCUSSION}

Our results demonstrate the natural course of SCI among those who have survived across the four decades since the inception of the study. Given the diminished life expectancy after $\mathrm{SCI},{ }^{4}$ these participants have had extraordinary survival. The findings should be used to help people understand the natural course of SCI and aging and to prepare for long-term maintenance of health, employment and quality of life after SCI.

Changes in health during the period of $30-40$ years after injury indicate a much more substantial and rapid health decline than that previously observed. The dramatic increase in the portion of individuals with $\geqslant 10$ non-routine physician visits and a significant upturn in the portion of individuals having a week or more of hospitalizations was consistent, with subjective declines in satisfaction with general health. Therefore, it appears that the changes in health may be affecting subjective well-being.

Consistent with earlier stages of the study, there was a somewhat mixed pattern of favorable and unfavorable changes over time, as compared with a simple, linear negative aging effect across all outcomes. Of particular interest was that changes in the three participation indicators did not change significantly, yet there were

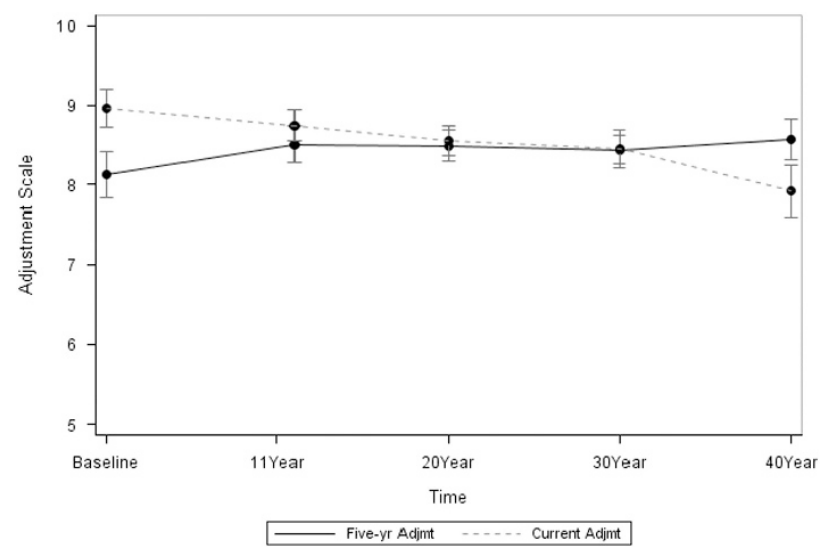

Figure 4 Current overall adjustment and expected adjustment in 5 years. A full color version of this figure is available at the Spinal Cord journal online.

declines in satisfaction with sex life and social life. Satisfaction with sex life actually plummeted between the 20 - and 30-year follow-ups, with little further change during the past decade of the study. Satisfaction with social life also declined over the last half of follow-up, but the decline was more gradual, decreasing between each of the last two 10 -year intervals. Taken together, this pattern suggests that declines in satisfaction with social life and sex life may actually occur earlier or in the absence of actual changes in overall indicators of participation, including either time out of bed or time away from home. This perhaps is related to the quality of these activities. For instance, individuals may get up and out of the house but with greater effort and less satisfaction. We cannot determine this from the current study design.

Not all areas of satisfaction declined, likely owing to resiliency among these long-term survivors. Satisfaction with employment actually increased during the first 10 years and remained stable thereafter, despite declines in the last decade in the actual portion of individuals employed and the number of hours working among those who are employed. The changes in participation and employment are expected given the age of the participants, and they have not led to corresponding declines in satisfaction with employment, finances or living arrangements. There may have been an expectation of employment changes and, therefore, corresponding maintenance of satisfaction with employment patterns over the years.

Some of the more intriguing findings were in self-rated current and predicted future adjustment. The lack of changes in self-ratings of current adjustment over time is somewhat surprising in that other outcomes were changing substantially, first improving over the first approximate 20 years of follow-up and declining thereafter. Yet, individuals viewed their ability to adjust to SCI as constant. Perhaps they attributed the changes in outcomes as owing to external factors. Given the length of survival of these participants, having a consistent view of one's adjustment over time, rather than one that changes with the ups and downs of life after SCI, may be a favorable characteristic that promotes survival.

In contrast, predicted future adjustment showed a much different pattern, declining gradually and steadily over time. These declines were not highly related to trends in other outcomes, as predicted future adjustment did not improve although other outcomes were improving, but rather it tended to decline regardless of whether other outcomes were changing. As individuals continue to survive and do very well in several life areas, including employment and home life, a lack of optimism about the future may not be a negative indicator, 
as one might believe. Considering both adjustment items together, it is ironic that, despite concerns about future adjustment, 5 years later the individual's ability to adjust continued to be stable.

Taken together, the findings demonstrate the ongoing resilience of those with exceptional survival in adapting psychologically to their situation. Although health has diminished for a significant portion of the participants and declines have been noted in satisfaction with sex life and social life for the last two decades, individuals continue to see themselves as well-adjusted and continue to be satisfied with multiple other areas of life. This is in spite of concerns about the future, as expressed in the changing ratings of future adjustment. This explanation is consistent with studies of resilience among those with physical disabilities. ${ }^{26}$ Resilience is protective, as greater resilience has been associated with lower reports of depressive symptoms, greater social functioning and greater satisfaction with life, findings that seem to remain consistent for individuals in acute rehabilitation and those with lengthy duration of disability. ${ }^{27,28}$ Research in individuals with physical disabilities has indicated greater resilience in older adults, ${ }^{29}$ paralleling research in older adults in the general population that suggests satisfaction with life despite age-related medical conditions and disability. ${ }^{30}$

One potential explanation for the spike in the need for physician visits and hospitalizations relates to expectation. As these individuals have remained generally healthy for decades and were able to maintain employment, they may be caught off guard by the dramatic changes in health and need for services. It is possible, if not probable, that many of the changes result from negative health spirals, some of which may have started with a single event. For instance, because of changes in skin resilience and skin breakdown, that previously may have been minor and quick to heal, may rapidly develop into life-changing or life-threatening health decline later in the aging process. Greater awareness of potential rapid declines may help individuals to prevent these changes from occurring. These mechanisms are speculative at this point but important to consider as we interpret the study findings.

Those wishing to develop interventions would be well advised to target interventions toward individuals with intermediate levels of aging, as the current changes in satisfaction with social life and sex life began about 20 years into the study or, on average, about 25 years after injury. If interventions occur only at later stages of life, it may be too late for successful prevention strategies, and prevention is always preferable to treatment. Any intervention must take into account the full pattern of changes in outcomes over time, as interpretation of isolated areas of life, such as predicted future adjustment, may present an inaccurate or even misleading overall picture of an individual's adaptation and likely future problems.

\section{Methodological considerations}

The primary strength of this study is unparalleled length of follow-up, with the same questions asked over time to the same individuals, all of whom have reached aging milestones. The corresponding limitation is that, as with all longitudinal studies and in particular those of populations with high mortality rates, attrition is high, and the overall sample size 40 years later is relatively small $(n=49)$. Although unparalleled in terms of the number of individuals with SCI reaching aging milestones, for statistical purposes, the sample size restricted our ability to control for other measures in the regression models. Similarly, the measurement is limited to a relatively small number of global indicators of important outcomes.

Sample size has been augmented and the scope of measurement expanded in later follow-ups, but these true longitudinal comparisons dating back to the study's initiation in 1973 are limited to repeat responders and to the variables included in the original assessment. We chose to use five of the eight times of follow-up measurement to consistently have approximate 10 -year intervals, rather than an initial 11 -year interval followed by 5 -year intervals. This not only kept the relative time frame between measurements consistent but also allowed us to maximize the overall sample size that would have been reduced by including each 5 -year data.

The data are limited to self-report. Therefore, there may be recall bias on variables asking individuals to recall events, such as the number of hospitalizations or physician visits. However, we kept the time frame relatively short ( 24 months) to limit the recall bias.

The study findings are generalizable to long-term survivors who are aging significantly with their SCI. Historically, the proportion of individuals living to these aging milestones is relatively small. Given the overall trend of decreasing satisfaction with sex life and social life mid-study, strategies should be developed to prepare individuals for these changes and perhaps circumvent them. Psychosocial interventions would clearly be appropriate at this point, perhaps even those that provide information on physiological changes or changing relationships. Also, the dramatic change in health outcomes needs to be accounted for in prevention strategies.

\section{Future research}

The current study measures only global indicators of health and need for services (physician visits and hospitalizations), so future research needs to focus more on the identification of the sequence of changes in health over relatively short periods of time. This is a complex challenge in longitudinal research, yet we clearly need to better understand how health declines so rapidly among those aging with SCI. We also need to address some of those factors that have been linked to successful aging in the general population, such as value change and spiritual growth. ${ }^{31} \mathrm{We}$ also need a better understanding of how interpersonal and social relationships change over time.

Quantitative methods alone are not going to answer all the remaining questions regarding aging and SCI. Qualitative studies are needed to have greater sensitivity to the perspectives of those who have reached aging milestones and to tie together what may otherwise seem like unique experiences. It is only through persistent efforts that we will understand better the issues of aging facing those reaching aging milestones after SCI and better prepare them for these changes.

\section{CONCLUSION}

Outcomes changed substantially over the 40-year time frame, although not at the same pace or necessarily in the same direction among these individuals with extraordinary survival. There were many declines that could be attributed to age, including diminishing satisfaction with sex life, social life and health, diminished optimism about future adjustment and a substantial increase in need for both non-routine physician visits and hospitalizations after 30 years of injury. On the other hand, employment outcomes only declined between the 30 - and 40-year follow-ups, which would be anticipated, as age-related changes and satisfaction with employment were maintained throughout. Individuals also maintained their satisfaction in living arrangements and finances while maintaining their overall level of adjustment.

\section{DATA ARCHIVING}

There were no data to deposit.

\section{CONFLICT OF INTEREST}

The authors declare no conflict of interest. 


\section{ACKNOWLEDGEMENTS}

The contents of this presentation were developed under grants from the US Department of Health and Human Services Administration for Community Living, NIDILRR grant numbers 90IF0015 (formerly H133G110157). NIDILRR is a Center within the Administration for Community Living (ACL), Department of Health and Human Services (HHS). The contents of this paper do not necessarily represent the policy of NIDILRR, ACL, HHS, and one should not assume endorsement by the Federal Government. We thank the following, without whom this publication would not have been possible: Richard Aust, Dr Yue Cao, Jennifer Coker, Dr Nicole DiPiro, Aaron Haas, Alex Jackson, Melinda Jarnecke, Laura Kline, Dr Chao Li, Kristian Manley, Alana McMichael, Karla Reed, Jameka Rembert, D’Andra Roper, and Dr Lee Saunders.

1 Devivo MJ. Epidemiology of traumatic spinal cord injury: trends and future implications. Spinal Cord 2012; 50: 365-372.

2 Strauss D, DeVivo MJ, Shavelle R. Long-term mortality risk after spinal cord injury J Insur Med 2000; 32: 11-16.

3 Putzke JD, Barrett JJ, Richards JS, DeVivo MJ. Age and spinal cord injury: an emphasis on outcomes among the elderly. J Spinal Cord Med 2003; 26: 37-44.

4 Middleton JW, Dayton A, Walsh J, Rutkowski SB, Leong G, Duong S. Life expectancy after spinal cord injury: a 50-year study. Spinal Cord 2012; 50: 803-811.

5 Groah SL, Charlifue S, Tate D, Jensen MP, Molton IR, Forchheimer M et al. Spinal cord injury and aging: challenges and recommendations for future research. Am J Phys Med Rehabil 2012; 91: 80-93.

6 Hitzig SL, Eng JJ, Miller WC, Sakakibara BM. An evidence-based review of aging of the body systems following spinal cord injury. Spinal Cord 2011; 49: 684-701.

7 Szlachcic Y, Adkins RH, Govindarajan S, Cao y, Krause JS. Cardimetabolic changes and disparities among persons with spinal cord injury: a 17-year cohort study. Top Spinal Cord Inj Rehabil 2014; 20: 96-104.

8 Charlifue SW, Weitzenkamp DA, Whiteneck GG. Longitudinal outcomes in spinal cord injury: aging, secondary conditions, and well-being. Arch Phys Med Rehabil 1999; 80: 1429-1434.

9 Weitzenkamp DA, Jones RH, Whiteneck GG, Young DA. Ageing with spinal cord injury: cross-sectional and longitudinal effects. Spinal Cord 2001; 39: 301-309.

10 Saunders LL, Krause JS, Focht KL. A longitudinal study of depression in survivors of spinal cord injury. Spinal Cord 2012; 50: 72-77.

11 Kennedy P, Kilvert A, Hasson L. A 21-year longitudinal analysis of impact, coping, and appraisals following spinal cord injury. Rehabil Psychol 2016; 61: 92-101.

12 Charlifue S, Gerhart K. Community integration in spinal cord injury of long duration. Neurorehabil 2004; 19: 92-101.
13 Amsters DI, Pershouse KJ, Price GL, Kendall MB. Long duration spinal cord injury: perceptions of functional change over time. Disabil Rehabil 2005; 27: 489-497.

14 Crewe N, Krause J. An eleven-year follow-up of adjustment to spinal cord injury. Rehabil Psychol 1990; 35: 205-210.

15 Krause JS, Crewe NM. Prediction of long-term survival of persons with spinal cord injury: an 11 year prospective study. Rehabil Psychol 1987; 32: 205-213.

16 Krause JS, Coker JL. Aging after spinal cord injury: a 30-year longitudinal study. J Spinal Cord Med 2006; 29: 371-376.

17 Krause JS, Broderick L. A 25-year longitudinal study of the natural course of aging after spinal cord injury. Spinal Cord 2005; 43: 349-356.

18 Krause JS. Changes in adjustment after spinal cord injury: a 20-year longitudinal study. Rehabil Psychol 1998; 43: 41-55.

19 Krause JS. Longitudinal changes in adjustment after spinal cord injury: a 15-year study. Arch Phys Med Rehabil 1992; 73: 564-568.

20 Krause JS, Bozard JL. Natural course of life changes after spinal cord injury: a 35-year longitudinal study. Spinal Cord 2012; 50: 227-231.

21 Krause JS. Survival following spinal cord injury: a fifteen-year prospective study. Rehabil Psychol 1991; 36: 89-98.

22 Krause JS, Kjorsvig JM. Mortality after spinal cord injury: a four-year prospective study. Arch Phys Med Rehabil 1992; 73: 558-563.

23 Krause JS, Crewe NM. Prediction of long term survival of persons with spinal cord injury: an 11 year prospective study. In: Eisenberg MG, Gluekauff RL (eds). Empirical Approaches to the Psychosocial Aspects of Disability. Springer: New York, USA, 1991.

24 Krause JS, Clark JM, Saunders LL. SCl longitudinal aging study: 40 years of research. Top Spinal Cord Inj Rehabil 2015; 21: 189-200.

25 Crewe NM, Athelstan GT, Bower A. Employment After Spinal Cord Injury: A Handbook for Counselors. University of Minnesota, Department of Physical Medicine and Rehabilitation: Minneapolis, MN, USA, 1978.

26 Quale AJ, Schanke AK. Resilience in the face of coping with a severe physical injury: a study of trajectories of adjustment in a rehabilitation setting. Rehabil Psychol 2010; 55: $12-22$.

27 White B, Driver S, Warren AM. Resilience and indicators of adjustment during rehabilitation from a spinal cord injury. Rehabil Psychol 2010; 55: 23-32.

28 Silverman AM, Molton IR, Alschuler KN, Ehde DM, Jensen MP. Resilience predicts functional outcomes in people aging with disability: a longitudinal investigation. Arch Phys Med Rehabil 2015; 96: 1262-1268.

29 Terrill AL, Molton IR, Ehde DM, Amtmann D, Bombardier C, Smith AE et al. Resilience, age, and perceived symptoms in persons with long-term physical disabilities. J Health Psychol 2014; 21: 640-649.

30 Montross LP, Depp C, Daly J, Reichstadt J, Golshan S, Moore D et al. Correlates of self-rated successful aging among community-dwelling older adults. Am J Geriatr Psychiatry 2006; 14: 43-51.

31 Vaillant GE. Aging Well: Surprising Guideposts to a Happier Life From the Landmark Harvard Study of Adult Development. Little, Brown: Boston, MA, USA, 2002. 